\title{
Thermal Relaxation in Perpendicular Recording with Single Pole Head and MR Head
}

\author{
W. H. Jiang, H. Muraoka, Y. Sugita and Y. Nakamura \\ RIEC, Tohoku Univ., 2-1-1, Katahira, Aoba-ku, Sendai 980-77, Japan
}

\begin{abstract}
Themal relaxation is becoming more and more significant as bit sizes are decreased to achieve high recording density. In this paper a separate read and write system, replacing the low read sensitivity single-pole head with a MR reproducing head and a regular SPT writing head, was used for experiments to measure the reproduced output reduction in double-layered media. The influence of the single-pole writing head during the reproducing process is brought out. Then, some simulation results associated with a curling switching model are also discussed.
\end{abstract}

Key word: Thermal relaxation, bit size, perpendicular recording, curling switching model

\section{INTRODUCTION}

It is well known that the magnetization of a particle surmounts an energy barrier and switches to an another stable state with the assistance of thermal energy, where the energy barrier and the thermal energy is given by $\mathrm{Ku} \cdot \mathrm{V}$ and $k T$ respectively ( $\mathrm{Ku}$ is the anisotropy constant and $\mathrm{V}$ is the particle volume, $k$ is Boltzmann's constant, $T$ is the absolute temperature). It has been reported that little time decay of output was measured using a MIG type ring head for recording/narrow gap ring head for readback in perpendicular recording[1]. On the other hand, a severe reduction of signal has also been reported[2]. In our experiments, a single-pole writing head and a highly sensitive MR reproducing head was used to measure the output time decay in double-layered media. Meanwhile it was also necessary to build up a correct simulation model to evaluate the particle reversal process, especially when the particle diameter decreases to an extremely small size. Here the media is assumed to be an assembly of separated, single domain, particulates and a curling switching model based on a 2-dimensional finite element method was introduced to calculate the time decay. The magnetic interaction among the particles, including exchange and magnetostatic interaction, was included by taking into account the mean field[3]. The mean field coefficient was determined by filting the observed $\mathrm{M}-\mathrm{H}$ loop with a simulated one. With this model the output reduction dependence on media parameters was simulated and compared with the experimental results.

\section{EXPERIMENTS}

In the experiments, we used a contact single-pole head for writing and a flying MR head for reproducing (flying height was $50 \mathrm{~nm}$ ) to measure the output reduction of the double-layered perpendicular media. To avoid any influence from the writing head during the reading process, the single-pole head was removed from media after writing and while reading with the MR head. Table 1 and Table 2 show the head and media parameters in our experiments respectively. The grain size of the media was estimated as $70 \mathrm{~nm}$ in diameter by AFM measurement.

Table 1 Head specification.

\begin{tabular}{|l|l|}
\hline Main pole material & Co-Zr-Nb amorplous \\
\hline Main pole thickness & $300 \mathrm{~nm}$ \\
\hline Track width & $50 \mu \mathrm{m}$ \\
\hline Number of turns & 26 \\
\hline
\end{tabular}

Table 2 Media specification

\begin{tabular}{|c|l|c|c|c|}
\hline \multicolumn{2}{|c|}{ Medium } & $\# \mathrm{~A}$ & $\# \mathrm{~B}$ & \#C \\
\hline Underlayer & Thickness $(\mu \mathrm{m})$ & 0.97 & 0.99 & 0.75 \\
\hline \multirow{4}{*}{$\mathrm{Co}-\mathrm{Cr}$} & Ms(emu/cm $\left.{ }^{3}\right)$ & 430 & 470 & 500 \\
\cline { 2 - 5 } & Thickness(nm) & 100 & 50 & 50 \\
\cline { 2 - 5 } & $\mathrm{Hc}(\mathrm{Oe})$ & 1100 & 1270 & 2000 \\
\cline { 2 - 5 } & $\Delta \mathrm{Hc} / \mathrm{Hc}$ & 0.61 & 0.86 & 0.1 \\
\hline
\end{tabular}

Figure 1 shows the recording density dependence of time decay for media whose thicknesses were $100 \mathrm{~nm}$ and $50 \mathrm{~mm}$. Over the measured time range almost no output reduction was apparant at $100 \mathrm{kFRPI}$. Even at a very low recording density of $5 \mathrm{kFRPI}$, where the demagnetization field is not sniall, the reduction was less than $3 \%$ for both media. This 
confirmed the advantage of low demagnetization fields at high recording density for perpendicular recording.

Next, the output reduction results for media with various coercive force deviation[4] is shown in Fig. 2. One thing which should be mentioned is that the value of coercive force of the two media was measured by different methods, one by VSM and another by Kerr effect measurement, so we think the values should be equal if measured by the same method. For a low coercive force deviation media, almost no output reduction was measured, even at a low recording density of 5kFRPI. This indicates that we should not only be concemed about the coercive force, but also its deviation when considering thermal relaxation.

\section{SIMULATION}

The grains of the media were assumed to be cylindrical with heights equal to the media thickness. Because the grain volumes of the media used in the experiments were relatively large, a curling switching model based on a 2dimensional finite element method was introduced to calculate the time decay for the smaller particle volumes used to achieve high recording density and $S / N$ ratio[5]. Table 3 gives the parameters used in the simulation. The mesh was built according to the single-pole head and double-layered perpendicular media used in the experiments. Table 4 shows the calculation process. Under a no relaxation condition, a recording process was simulated at one density, then the demagnetization field, calculated according to the original magnetization, was subtracted from the coercivity to determine the reverse field of the grain. Here $\mathrm{Ku}$ was calculated from the coercivity Hc, which is equal to the grain reverse field, instead of the anisotropy field $\mathrm{Hk}$ in the $\mathrm{S}-\mathrm{W}$ model. Thus, the time constant $\tau\left(=10^{-9}(\mathrm{Ku} \cdot \mathrm{V} / \mathrm{kT})\right)$ was determined. Following a time step $\Delta \mathrm{t}$, the magnetization was calculated by a selfconsistent calculation, according to the time decay defined as $M\left(t_{n}\right)=M\left(t_{n-1}\right) \times \exp \left(-\left(-t_{n-1}+\Delta t\right) / \tau\right)$.

The volume distributions must also be considered when we discuss the particle volume dependence. Fig. 3 shows time decay for various recording densities and volume distributions in which $\mu$ is the average particle volume $\left(7.0 \times 10^{4} \mathrm{~nm}^{3}\right)$ and $\sigma$ is the standard deviation of

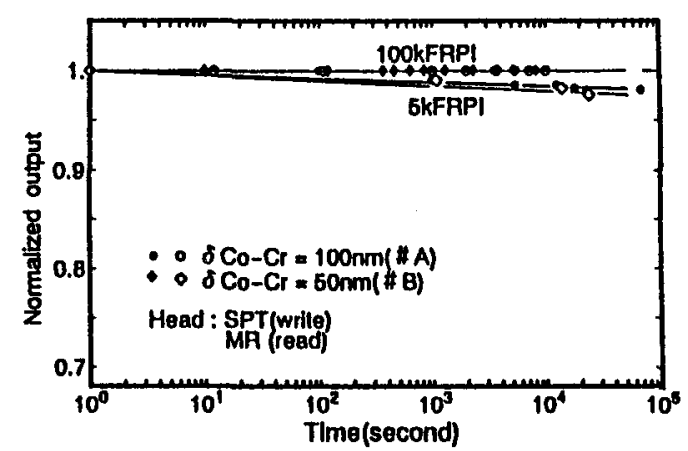

Fig.1 Dependence of time decay on recording densities

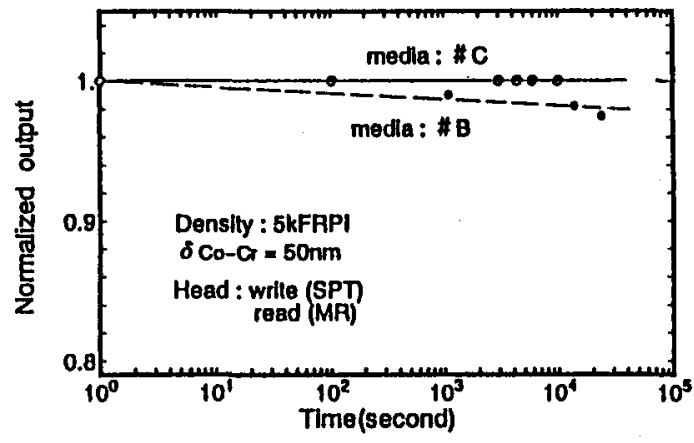

Fig. 2 Time decay dependence on coercive force deviation Table 3 Medium specification for the simulation

\begin{tabular}{|l|l|}
\hline Saturation magnetization & $430 \mathrm{emu} / \mathrm{cm}^{3}$ \\
\hline Anisotropy field & $4,360 \mathrm{Oe}$ \\
\hline Anisotropy field distribution & 0.21 \\
\hline Cocrcive force & $1,100 \mathrm{Oe}$ \\
\hline Thickness & $100 \mathrm{~nm}$ \\
\hline Menn volume of particles & $70,000 \mathrm{~nm}^{3}$ \\
\hline Temperalure & $293 \mathrm{~K}$ \\
\hline
\end{tabular}

Table 4 Simulation flow chart

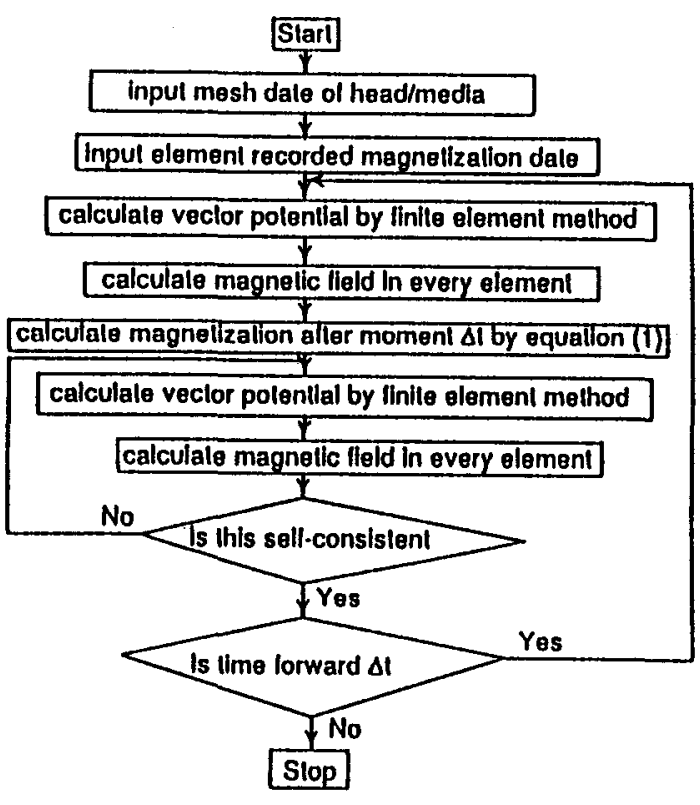

$M(\ln )=M(\ln \cdot 1) \times \exp (-(\ln \cdot 1+\Delta 1) / \tau)$

(I) 
volume. For a small $\sigma / \mu(=0.1)$, there was almost no output reduction at both high and low recording density. The severe output reduction at large $\sigma / \mu$ confirms that the relaxation of grains whose volumes are smaller than the average dominates the time dependence. The same simulation was done for a medium whose thickness was $50 \mathrm{~nm}$ and is shown in Fig. 4. Compared with the results for the $100 \mathrm{~nm}$ medium there was almost no change for small $\sigma / \mu(=0.1,0.2)$, but a rapid decrease for $\sigma / \mu(=0.3)$. The small output reduction at high recording density confirms the low demagnetization character of perpendicular recording at high density, which is also proven by experiment.

Figure 5 shows the reduction in output as the particle diameter is decreased. The film thickness is $50 \mathrm{~nm}$. For a large particle diameter $(D=70 \mathrm{~nm})$, there was almost no output reduction, fitting well with the experiment. Decreasing the diameter to $16 \mathrm{~nm}$, with a small volume distribution( $\sigma / \mu=0.1$, the output reduces to $84 \%$ after 10 years, but for $\sigma / \mu=0.2$, the decrease is much quicker. The ratio $\mathrm{Ku} \cdot \mathrm{V} / \mathrm{kT}$ is 114 when the particle diameter is $16 \mathrm{~nm}$. Thus, we can reduce thermal relaxation in perpendicular recording media with relatively small particles and achieve high recording density and low noise because of the large film thickness.

Figure 6 shows the dependence of output reduction on temperature. The temperature dependence of media cocrcivity is neglected. At a relatively small particle volume of $1.0 \times 10^{4} \mathrm{~nm}^{3}$ and with $\sigma / \mu=0.1$, when the absolute temperature rises to $373 \mathrm{~K}$, the output will decrease from $88 \%$ at $293 \mathrm{~K}$ to $75 \%$ after 10 years at a density of 130kFRPI.

\section{INFLUENCE OF SINGLE-POLE WRITING HEAD}

We discussed the time decay in perpendicular doublelayered media by using a single-pole writing head and a flying MR reproducing head above. But when using singlepole head for writing and reading, even the slight influence of the head gave rise to a remarkable measurement error for thermal relaxation. In order to confirm the influence of single-pole head, the following experinent was preformed.

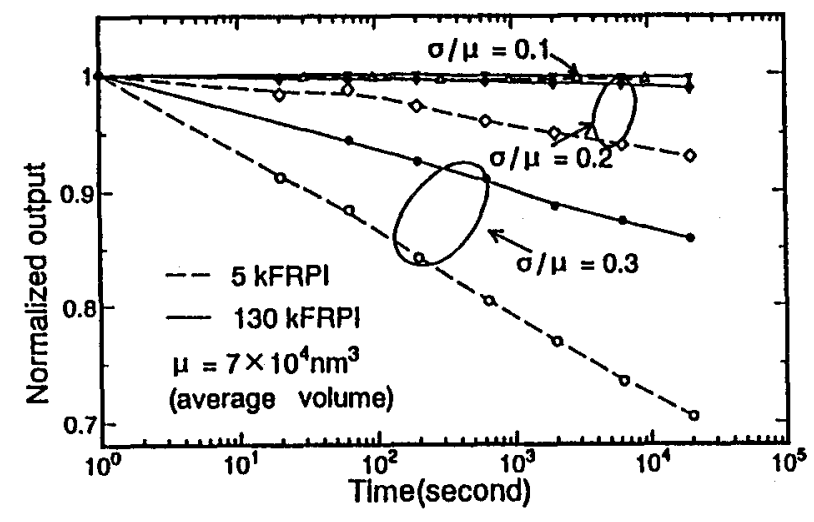

Fig.3 Time decay at various recording densities and volume deviations (media thickness is $100 \mathrm{~nm}$ )

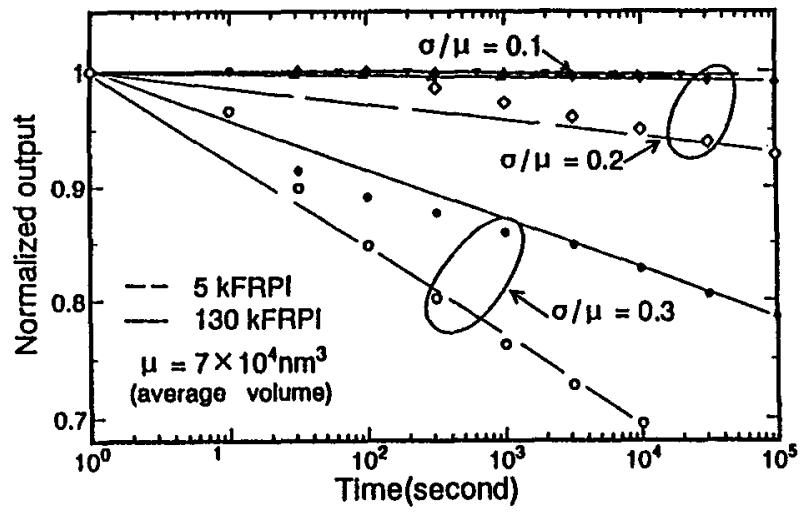

Fig. 4 Time decay at various recording densities and volume deviations (media thickness is $50 \mathrm{~nm}$ )

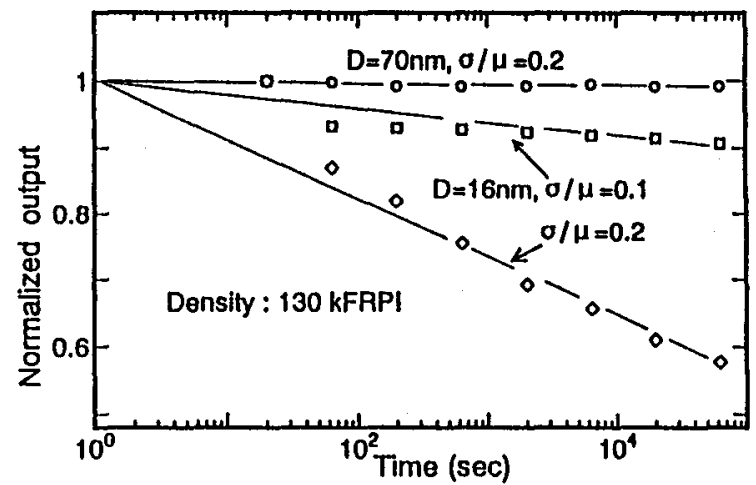

Fig.5 Dependence of time decay on particle diameters

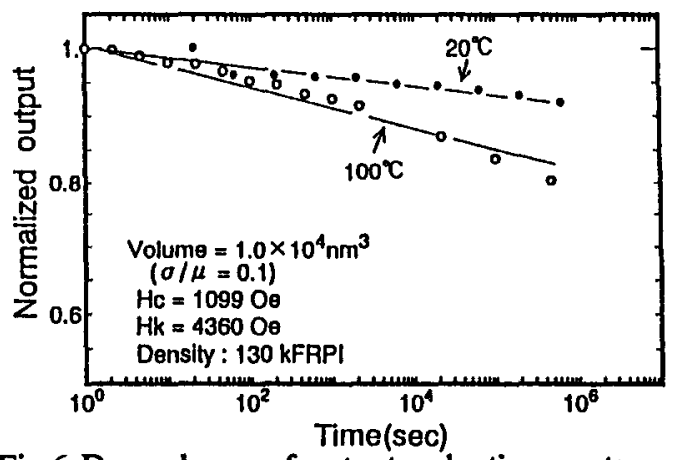

Fig.6 Dependence of output reduction on temperature 
Figure 7 illustrates the results when the single-pole head is continuously in contact with the media during the whole reading process when reading by an MR head, then compares this with the results for the case with the singlepole head removed after writing. A large output reduction was measured when the single-pole head was in continuous contact. We suggest that for a low anisotropy, soft film head, some external field may be absorbed in the film, or the head domain struclure may be changed during the reading process and this would destroy the remanence of the media. For a high anisotropy-field head, the domain structures are somewhat stable, so heads with various Hk were used to measure the time decay. Figure 8 shows the results. The output reduction was reduced to less than $3 \%$ after two hours by increasing $\mathrm{Hk}$ to $14.80 \mathrm{e}$ at a recording density of $110 \mathrm{kFRPI}$. This implies that improved head Hk is somewhat effective for decreasing the head influence itself.

\section{CONCLUSION}

We used a regular SPT writing head and a flying MR reproducing head to measure the time decay and discussed the influence of the single-pole head during the reading process in perpendicular double-layered media. The output reduction was also calculated for extremely small particle diameters. The low demagnetization field character of perpendicular recording at high recording density was proven by experiments and simulation. We can make the thermal relaxation small with relatively small particles to achieve high recording density and low noise while maintaining the large film thickness of perpendicular recording media. However, this requires a small particle volume and narrow coercivity distribution and the influence of domain instability in the single-pole head must also be carefully considered.

\section{ACKNOWLEDGEMENTS}

The authors wish to express their gratitude to Master degree student $\mathrm{K}$. Sato and Mr. T. Katakura and Mr . K. Yazawa in SONY Corp. for supplying the thin-film head and Mr.I.Tagawa who is now at Fujitsu Ltd. for the

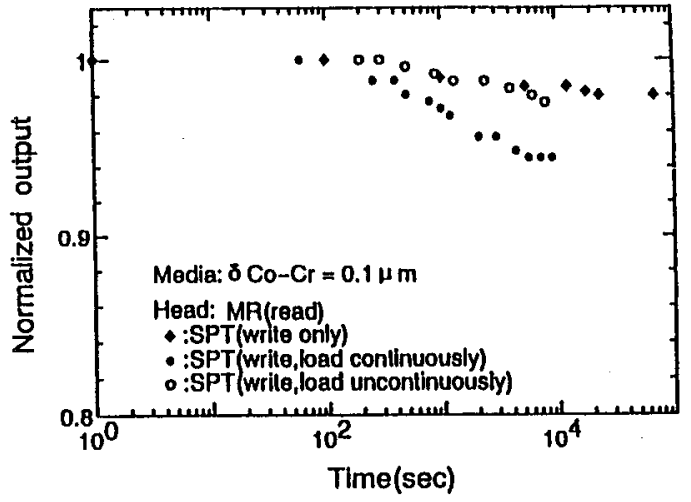

Fig.7 Influence of single-pole head

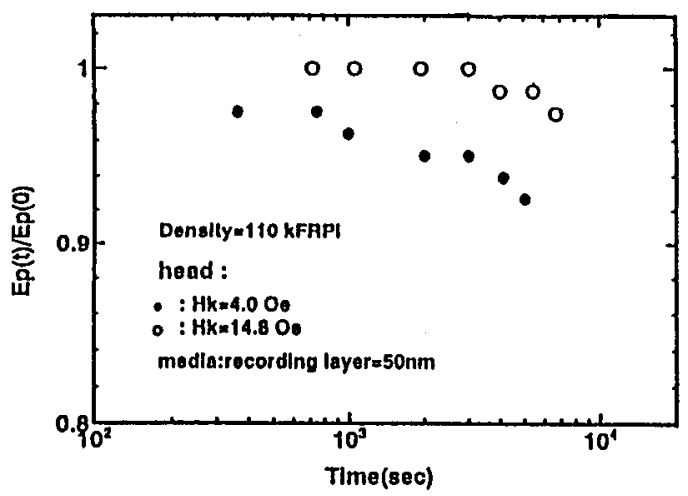

Fig.8 Output reduction for various head $\mathrm{Hk}$

simulation program. This research is partly supported by ASET and SRC.

\section{REFERENCE}

[1]N. Honda, K. Ouchi and S. Iwasaki, "1.5GBit/in ${ }^{2}$ Perpendicular Recording With Inductive Head," Intermag'96, GB-09 (1996)

[2]W. Cain, A Payne, M. baldwin and R. Hempstead, "Challanges in the Pratical Implementation of Perpendicular Magnetic Recording,"IEEE Trans. Magn.,Vol.32, p97(1996) [3]I. Tagawa, A. Takeo and Y.Nakamura, "Interparticle Interaction Field in Magnetic Recording Media," J. Magn. Soc. Jpn., Vol.18, p95-98 (1994) (in Japanese)

[4]I. Tagawa, Y. Nakamura, "Relationship Between High Density Recording Performance and Particle Coercivity Distribution,"IEEE Trans. Magn.,Vol.27,p4975-4978 (1991) [5]W.H. Jiang, H. Muraoka, Y. Tagawa and Y. Nakamura, "Bit Density Dependence of Thernal Relaxation in Perpendicular Magnetic Recording," J. Magn. Soc. Jpn., Vol.21, p293-295 (1997) (in Japanese) 